\begin{tabular}{|c|c|}
\hline ב & $\begin{array}{c}\text { International Journal of Current Research } \\
\text { and Academic Review }\end{array}$ \\
\hline $\begin{array}{l}\text { EXCELLENT } \\
\text { PUBLISHERS }\end{array}$ & $\begin{array}{c}\text { ISSN: 2347-3215 (Online) Volume } 6 \text { Number } 7 \text { (July-2018) } \\
\text { Journal homepage: http://www.ijcrar.com }\end{array}$ \\
\hline
\end{tabular}

doi: https://doi.org/10.20546/ijcrar.2018.607.003

\title{
Synthesis of Gold Nanoparticles Using Penicillium sp. and their Antibacterial Activity against Human Pathogens
}

\author{
R. Nigar Sulthana* and A. Rajanikanth \\ Department of Biotechnology, SPW Degree \& PG College, Tirupati, A.P, India \\ *Corresponding author
}

\begin{abstract}
This study was planned to obtain fungi from garden of SPW Degree and PG College, Tirupati with the extreme goal of producing antibacterial nanoparticles. Among 5 funguses isolated, an isolate coded as SPW-1 identified as Penicillium sp has produced nanoparticles extracellularly within $12 \mathrm{~h}$. These nanoparticles were characterized by UV-Vis. Spectrophotometer, TEM, and FTIR analysis. Penicillium sp synthesized gold nanoparticles showed many interesting morphologies with a size of $4-20 \mathrm{~nm}$. The presence and binding of proteins with nanoparticles was confirmed by FTIR study. Interestingly, the fungal derived gold nanoparticles exhibited superior antibacterial activity than the standard antibiotic, penicillin-G against E. coli and Bacillus cereus. Thus, the obtained results reveal that these antibacterial nanoparticles could be explored as promising candidates for a variety of biomedical and pharmaceutical applications.
\end{abstract}

\section{Article Info}

Accepted: 04 June 2018

Available Online: 10 July 2018

\section{Keywords}

Soil fungi, Penicillium Sp, Gold nanoparticles (GNPs), Antibacterial activity

\section{Introduction}

In the present day's bio synthesis of gold nanoparticles is one of the most active areas of research in the field of nanotechnology because metal nanoparticles are produced mainly by means of chemical processes that are toxic to the environment. Therefore a growing demand has been accumulated to develop environment friendly approaches to synthesize nanomaterials (Achintya Mohan Goswami and Sanjay Ghosh, 2013).

Naresh Niranjan Dhanasekar et al., (2015) in their research work said that microbes such as bacteria, fungi, diatoms, yeast and actinomycetes are used for the production of a large number of inorganic and heavy metal nanoparticles. However, the production of large biomass, easy handling/bioavailability, high metal tolerance, mineral solubilising activity, and less time make fungi extremely superior over other microbial resources. In addition, the studies on fungi can be easily extrapolated to others. The biosynthesis of nanoparticles through microbes primarily fungi, has been carried out both intracellularly and extracellularly (Vijayakumar and Prasad, 2009; Mukherjee et al., 2002). However, owing to the easy downstream processing and costeffectiveness, extracellular synthesis finds more extensive applications in industries than the intracellular route. Mukherjee et al., (2002) have been able to produce gold nanoparticles of various morphologies through incubation of fungal extract with $10^{-3} \mathrm{M} \mathrm{AuCl}_{4}{ }^{-}$in the dark. In the present study, we report the use of cell free filtrate of filamentous fungus Penicillium sp. for the synthesis of gold nanoparticles. The methodology adopted here is a simple, feasible, and single-step 
process, which does not require the usual usage of toxic and hazardous chemicals.

\section{Materials and Methods}

\section{Sample collection}

Soil samples were collected from garden of SPW Degree and PG College, Tirupati and were brought to the laboratory in sterile polythene bags and stored in refrigerator at $4^{\circ} \mathrm{C}$ for further use.

\section{Isolation and identification of fungal strains}

The Fungal strains isolated by serial dilution of $1.0 \mathrm{~g}$ garden soil and pure cultures of isolates were made by Streak-plate method on Potato Dextrose Agar (PDA) medium. The isolated fungal strains taxonomically identified by lacto phenol cotton blue method (Aneja Lab Manual 2004).

\section{Screening of soil fungi for mycogenic synthesis of gold nanoparticles}

All the isolates were screened for the synthesis of gold nanoparticles. To prepare the biomass, the fungi were grown aerobically in potato dextrose broth (PDB) and were incubated at $28^{\circ} \mathrm{C}$ for 7 days. After incubation, fungal mat was washed with sterile distilled water. Typically, $10 \mathrm{~g}$ (wet weight) of fungal mat was brought into $100 \mathrm{~mL}$ sterile distilled water in an Erlen Meyer flask and was kept under shaker condition (120 rpm) for $48 \mathrm{~h}$ at $28^{\circ} \mathrm{C}$. Then, the mycelial free filtrate was obtained by passing it through Whatman filter paper No.1. The filtrate was reacted with known quantity of gold chloride ion solution to yield an overall $\mathrm{Au}+$ ion concentration of $10^{-3} \mathrm{M}$ and the reaction was carried out in dark at room temperature. Concurrently, the mycelial free extract and gold chloride solution were maintained as controls and the change in colour was observed up to 48 h (Balakumaran et al., 2016).

\section{Characterization of mycosynthesized gold nanoparticles}

Preliminary characterization of gold nanoparticles was done through visual observation for change in colour from pale yellow to a violet. Time dependent formation of gold nanoparticles was observed using UV-vis. Spectrophotometer. The mycosynthesized silver nanoparticles was confirmed by sampling the reaction mixture at regular intervals and the absorption spectra was scanned at the wavelength of $300-700 \mathrm{~nm}$ in UV1800 spectrophotometer. For Fourier transform infrared spectroscopic (FTIR) analysis, the mycosynthesized gold nanoparticles were freeze-dried and then diluted with potassium bromide in the ratio of 1:100. The FTIR spectrum was recorded using Alpha FTIR instrument. For electron microscopic studies, $10 \mu \mathrm{L}$ of mycosynthesized silver nanoparticles was drop coated on carbon grid and the images of nanoparticles were studied using transmission electron microscopy (TEM) (Alaa A. A. Aljabali et al., 2018).

\section{Antibacterial activity of mycoderived gold nanoparticles}

In this study, two different bacteria $E$. coli and Bacillus cereus were used as test organisms and grown in nutrient agar medium. Well diffusion assay was performed to determine the antibacterial activity of mycosynthesized gold nanoparticles. Different concentrations of $(25 \mu \mathrm{L}$ to $100 \mu \mathrm{L}$ ) gold nanoparticles were loaded separately into each well of the Petri plates. Mycelial free extract was used to compare the antibacterial activity of synthesized nanoparticles; also Penicillin-G $(1 \mathrm{mg} / \mathrm{mL})$ was used as a positive control. After inoculation, the plates were incubated at $37^{\circ} \mathrm{C}$ for $24 \mathrm{~h}$ and the zone of inhibition (ZOI) was measured in terms of millimetre. These assays were carried out in triplicate.

\section{Results and Discussion}

\section{Isolation and identification of fungal strains}

Two soil samples were collected from garden of SPW Degree and PG College, Tirupati. A total of 5 isolates were isolated from collected samples and were named as SPW1-SPW5. All of these isolates were screened for gold nano particle synthesis. Among the screened fungi, based on visual observation, the fungus SPW1 found to be effective in the synthesis of gold nano particles when compared to other species. This fungus SPW1 after staining with lacto phenol cotton blue and using taxonomic with microscopic observation was identified as Pencillium sp (Figure 1).

\section{Characterization of mycosynthesized gold nanoparticles}

In the present study, extracellular mycosynthesis of AuNPs was carried out by using the fungal filtrate and the conversion of gold ions $(\mathrm{Au}+)$ to elemental gold (Auo) was investigated by visual observations (Figure 2 ). 
Fungi were grown aerobically in MYPG broth medium to obtain the fungal biomass. Suspending the fungal biomass in distilled water for 3 days under stationary conditions allowed the diffusion of some cellular substances, such as reductase enzymes or other reducing substances, outside the cell. These cellular substances can then interact with gold chloride ions, reducing them to gold atoms, forming nuclei that aggregate to give $\mathrm{Au}-$ NPs. The addition of gold chloride solution to the fungal filtrate resulted in the abrupt change in the colour of the filtrate from pale yellow to a faint purple colour. The colour change was due to the excitation of surface Plasmon vibrations, which is a characteristic feature of synthesized nanoparticles (Song et al., 2009).

The UV-Vis spectra were recorded as shown in figure 3, for $1 \mathrm{mM}$ gold chloride tetra hydrate salt solution and reaction mixture containing cell free growth medium and $1 \mathrm{mM}$ gold chloride tetrahydrate salt solution. The light absorption patterns were monitored in the range of 300$700 \mathrm{~nm}$. Long term incubation carried out for $96 \mathrm{hr}$ of reaction mixtures resulted in the spectra of increasing intensity where major peak of gold was observed in the range 500-600 $\mathrm{nm}$. During this incubation, UV-visible spectrum of the medium was recorded to study the change in light absorption profile of the medium due to change in intensity of the colour change. The UV-visible spectra of gold were recorded at various time intervals showed increased absorbance with increasing time of incubation at around $552 \mathrm{~nm}$. While the control gold chloride solutions do not show any obvious peaks.

Fourier transform infrared spectroscopy analysis enabled the identification of potential functional groups present on the surface. FTIR spectra of the fungal extract and synthesized GNP are shown in Figure 4. It indicates the disappearance as well as appearance of new peaks after synthesis of nanoparticles. FTIR spectra showed a broad contour in the range of $3600-3220 \mathrm{~cm}^{-1}$, which indicates the presence of -OH groups. The peak positioned at 2930 $\mathrm{cm}^{-1}$ could be attributed to the -CH stretch of aldehydes. The disappearance of this peak signifies their active participation in reduction of gold ions. After GNP synthesis, appearance of peaks at 1623 and $1355 \mathrm{~cm}^{-1}$ is attributed to the $\mathrm{C}-\mathrm{N}$ stretch of aliphatic amine. FTIR spectra show disappearance of peaks at $1000 \mathrm{~cm}^{-1}$ and $500 \mathrm{~cm}^{-1}$ attributed to bending vibration of $-\mathrm{CH}$ and $-\mathrm{NH}$ groups of amines. Similarly disappearance of peak at 683 $\mathrm{cm}^{-1}$ is due to disulphides group indicating its involvement in GNP formation (Ahmad et al., 2003). Complete absence of these bands after reduction signifies that amino acids such as cysteine and methionine may be involved in the reduction and/or stabilization process. The involvement of these functional groups strongly suggests the role of protein molecules in the bioreductive synthesis of GNP.

Fig.1 Morphology of the selected fungal isolate

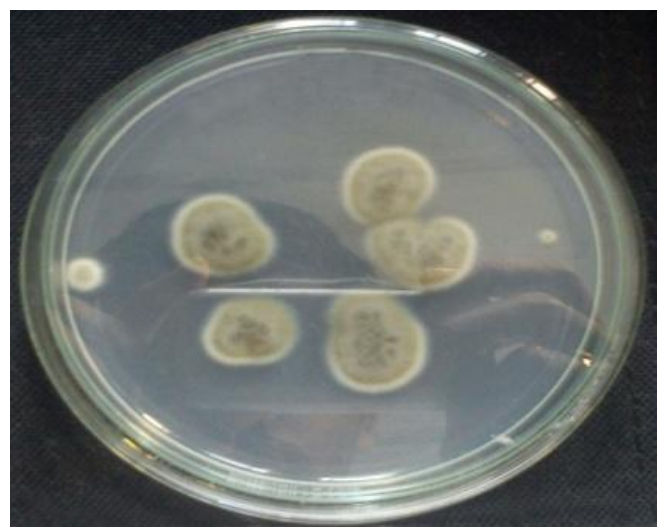

Fig.2 Visual observation of colour change in gold nanoparticles synthesis

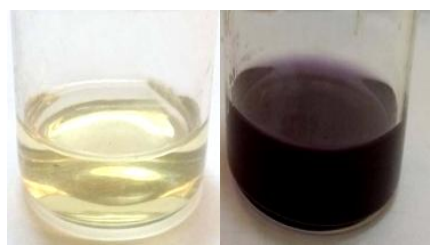


Fig.3 UV-Vis spectral analysis of synthesized gold nano particles

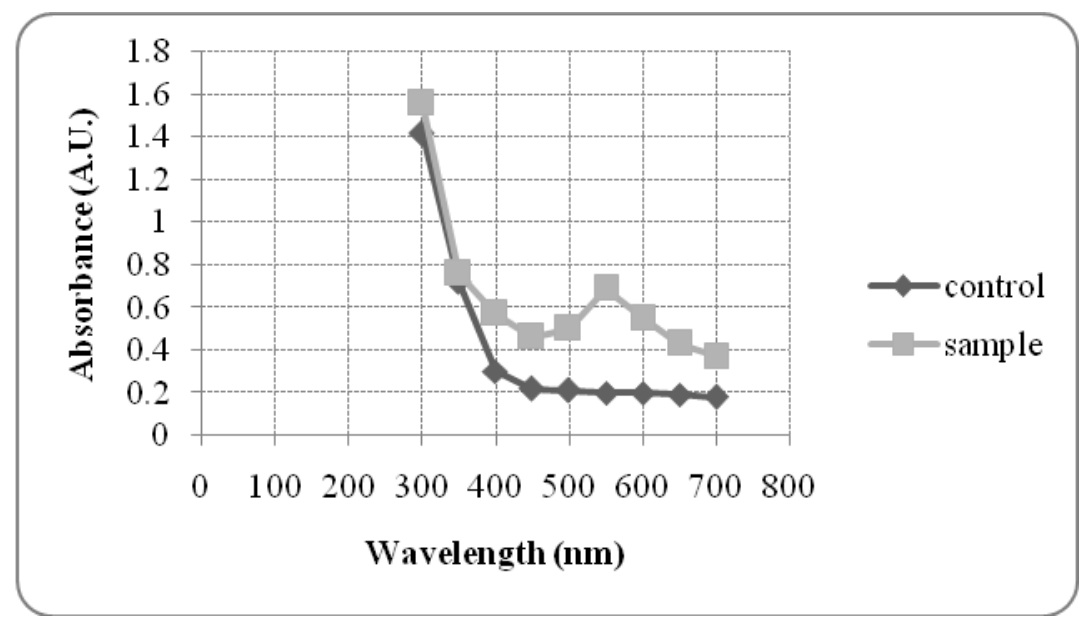

Fig.4 FTIR spectrum of the gold nanoparticles obtained using a cell-free filtrate of the fungus Penicillium sp.

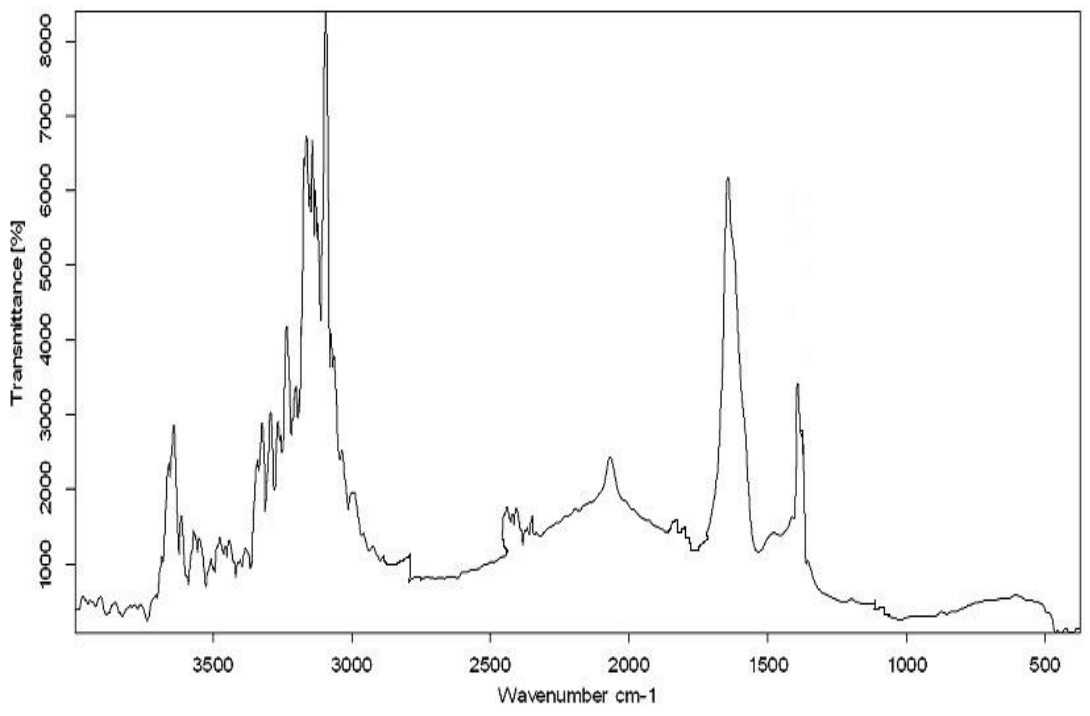

Fig.5 TEM micrographs of synthesized spherical shaped silver nanoparticles

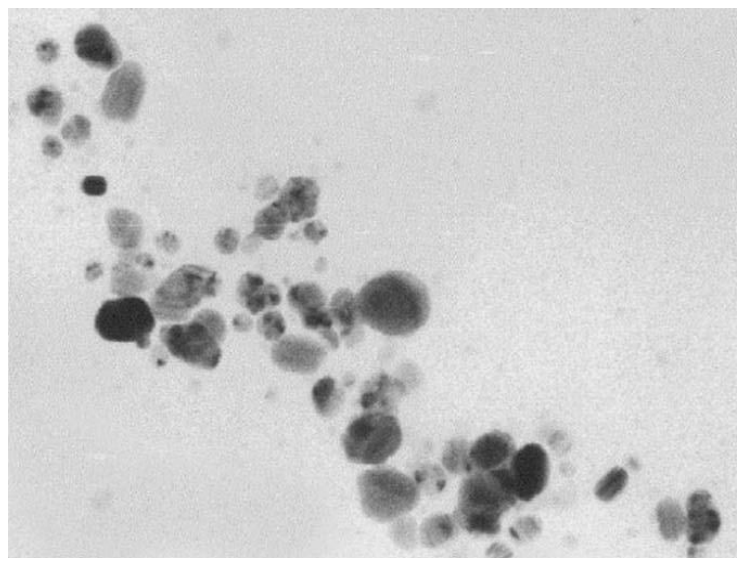


Fig.6 Zone of inhibition of green synthesized AuNPs against human pathogenic bacteria

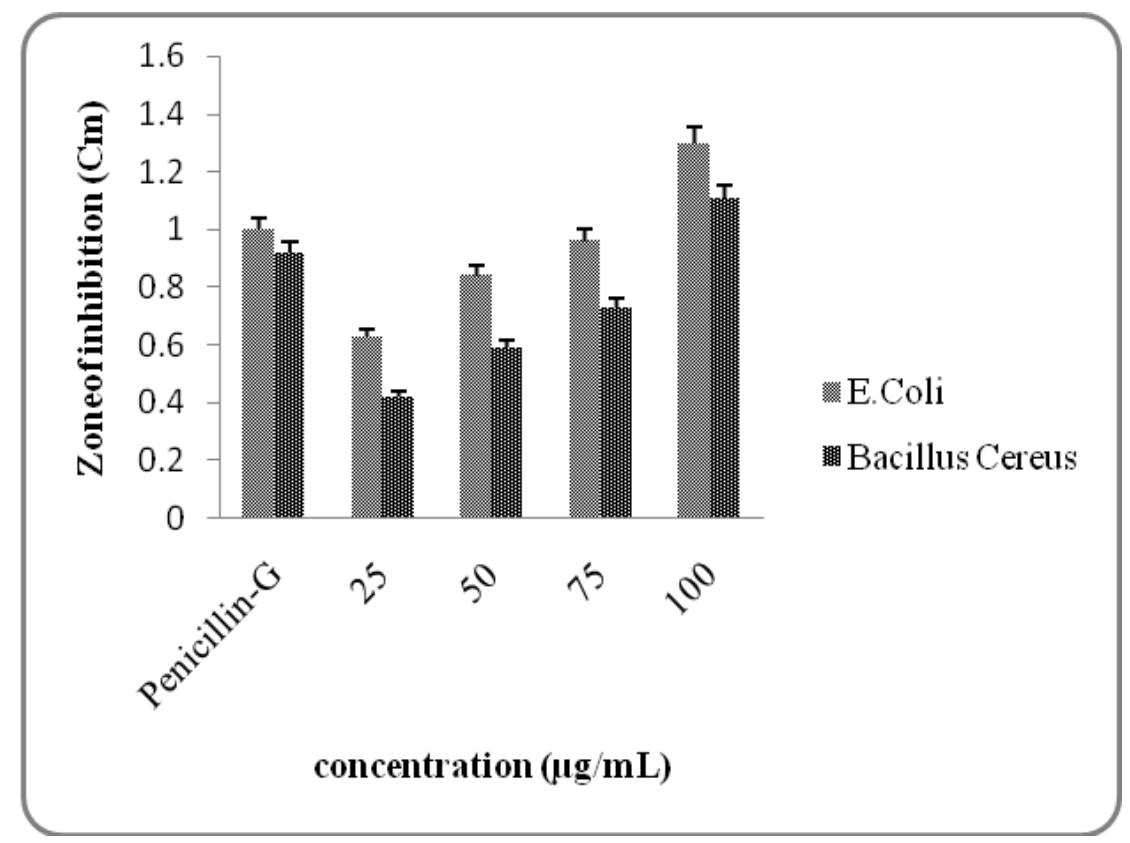

Transmission electron microscopy gives complete information regarding the size and morphology of the synthesized gold nanoparticles. Figure 5 represents the TEM images of gold nanoparticles synthesized using gold chloride with the cell-free filtrate of the filamentous fungus Penicillium sp. The representative TEM micrographs of AuNPs obtained after $96 \mathrm{hr}$ of incubation exhibited NPs with variable shapes; however, most of them were spherical Au-NPs with the majority having the size range of 4-20 nm. Similarly synthesis of spherical gold nanoparticles (GNPs) has been reported using the fungus Fusarium oxysporum (Mukherjee et al., 2002).

\section{Anti-bacterial activity of gold nanoparticles}

Toxicity of nanoparticles against pathogenic microorganisms has made them evolve as potential agents in clinical applications. Considering the fact that biosynthesized GNP exhibited an excellent antibacterial activity against both gram positive and gram negative bacteria. The biosynthesized GNP showed a distinct inhibition zone of $1.3 \mathrm{~cm}$ against the gram positive bacteria, and it was little effective against the gram negative bacteria (Figure 6). GNPs inhibit bacterial growth by generating holes in the cell wall, resulting in release of cell contents, or they bind with the DNA inhibiting its uncoiling thereby transcription (Rai et al., 2010). However, in the present case the probable reason behind the selective antibacterial activity might be the external structural differences between gram positive and negative bacteria (Kim et al., 2009; Priyadarshini et al., 2014).

In this study gold nanoparticles were synthesized by a potential fungal strain Pencillium sp. isolated from soil. The nanoparticles synthesized were characterized by UV, TEM, and FTIR analysis. The antibacterial efficacy of gold nanoparticles was tested against gram positive and gram negative pathogenic bacterial strains. We conclude that physical and chemical synthesis of GNPs are found to be expensive and there may be effect to GNPs by various toxic chemicals, whereas biological synthesis is the more preferred option for nanoparticles with high efficacy of antibacterial activity.

\section{References}

Achintya Mohan Goswami, Sanjay Ghosh. Biological Synthesis of Colloidal Gold Nanoprisms Using Penicillium citrinum MTCC9999. Journal of Biomaterials and Nanobiotechnology; 2013, 4: 2027.

Ahmad A, Senapati S, Khan M.I, Kumar R, Sastry M. Extracellular biosynthesis of monodisperse gold nanoparticles by a novel extremophilic Actinomycete, Thermomonospora sp. Langmuir; 2003, 19(8): 3550-3553.

Alaa A. A, Aljabali, Yazan Akkam, Mazhar Salim Al Zoubi, Khalid M. Al-Batayneh, Bahaa Al-Trad, 
Osama Abo Alrob, Alaaldin M. Alkilany, Mourad Benamara, David J. Evans. Synthesis of Gold Nanoparticles Using Leaf Extract of Ziziphus zizyphus and their Antimicrobial Activity. Nanomaterials; 2018, 8: 174.

Aneja K.R. Experiments in microbiology plant pathology and biotechnology, 4th edn. New Age International (P) Ltd., Publishers, New Delhi, 2004; p 320.

Balakumarana M.D., Ramachandrana R, Balashanmugama P, Mukeshkumarb D.J, Kalaichelvan P.T. Mycosynthesis of silver and gold nanoparticles: Optimization, characterization and antimicrobial activity against human pathogens. Microbiological Research; 2016, 182: 8-20.

Kim J, Lee J, Kwon S, Jeong S. Preparation of biodegradable polymer/silver nanoparticles composite and its antibacterial efficacy. Journal of Nanoscience and Nanotechnology; 2009,

Malvia S, Bagadi SA, Dubey US, Saxena S. Epidemiology of breast cancer in Indian women. Asia Pac J Clin Oncol; 2017 Aug; 13(4): 289-295.

Mukherjee P, Senapati S, Mandal D, Ahmad A, Khan M.I, Kumar R, Sastry M. Extracellular Synthesis of Gold Nanoparticles by the Fungus Fusarium oxysporum. Chembiochem; 2002, 3 (5): 461-463.

\section{How to cite this article:}

Nigar Sulthana R. and Rajanikanth A. 2018. Synthesis of Gold Nanoparticles Using Penicillium sp. and their Antibacterial Activity against Human Pathogens. Int.J.Curr.Res.Aca.Rev. 6(7), 19-24.

doi: $\underline{\text { https://doi.org/10.20546/ijcrar.2018.607.003 }}$
Naresh Niranjan Dhanasekar, Ganga Ravindran Rahul, Kannan Badri Narayanan, Gurusamy Raman, Natarajan Sakthivel. Green Chemistry Approach for the Synthesis of Gold Nanoparticles Using the Fungus Alternaria sp. Journal of Microbiology and Biotechnology; 2015, 25(7): 1129-1135.

Priyadarshini E, Pradhan N, Sukla L.B, Pand P.K. Controlled synthesis of gold nanoparticles using Aspergillus terreus IF0 and its antibacterial potential against Gram negative pathogenic bacteria. Nanotechnology Journal; 2014,

Rai A, Prabhune A, Perry C.C. Antibiotic mediated synthesis of gold nanoparticles with potent antimicrobial activity and their application in antimicrobial coatings. Journal of Materials Chemistry; 2010, 20(32): 6789-6798.

Song J.Y, Jang H.K, Kim B.S. Biological synthesis of gold nanoparticles using Magnolia kobus and Diopyros kaki leaf extracts. Process Biochemistry; 2009, 44: 1133-1138.

Vijayakumar P.S, Prasad B.L.V. Intracellular biogenic silver nanoparticles for the generation of carbon supported antiviral and sustained bactericidal agents. Langmuir; 2009, 25: 11741-11747. 